\title{
Novel Titanium Dioxide Based Nanocomposite Anodes for Li-Ion Batteries
}

\author{
M. Oguz Guler*, O. Cevher, U. Tocoglu, T. Cetinkaya and H. Akbulut \\ Sakarya University, Engineering Faculty, Metallurgical \& Materials Engineering Department \\ Esentepe Campus, 54187, Serdivan/Sakarya, Turkey
}

\begin{abstract}
Carbon nanotube thin sheets - buckypapers - were prepared from multi-walled carbon nanotubes oxidized with different oxidation agents. Titanium dioxide films were then deposited by thermal evaporation of Ti films on buckypapers substrates followed by in situ dc plasma oxidation. The effect of oxidation power on the structural, compositional and electrochemical properties of the films was investigated.
\end{abstract}

DOI: 10.12693/APhysPolA.123.390

PACS: 61.48.De, 82.45.Mp, 82.47.-a

\section{Introduction}

The graphite anode electrode in commercial lithium ion batteries has several disadvantages such as its electrical disconnection, structural deformation, and initial loss of capacity [1-3]. Especially, $\mathrm{TiO}_{2}$ is regarded as a promising active lithium intercalation material with low production cost and high capacity, low-voltage (below ca. $2.0 \mathrm{~V} \mathrm{vs.} \mathrm{Li}^{+} / \mathrm{Li}$ ) for lithium intercalation. More recently, much attention has been paid to fabricate $\mathrm{TiO}_{2}$-based hybrid nanostructures as anode materials with enhanced lithium storage performance. One of the strategy is to introduce a buffering matrix that can prevent the breaking down of $\mathrm{TiO}_{2}$-based hybrid nanostructures during cycling. Since the landmark paper by Iijima [4], carbon nanotubes (CNTs) have attracted considerable research interest due to their unique combination of mechanical, electrical and thermal properties. Forms of CNTs in the macroscopic level, such as forests, yarns and films have been reported providing a practical venue to utilize and manipulate the remarkable properties of CNTs for broad applications [5, 6]. Paper-like CNT films, also called buckypapers, are self-supporting networks of entangled CNT assemblies arranged in a random fashion and held together by van der Waals interactions at the tube-tube junctions.

In this study, CNTs- $\mathrm{TiO}_{2}$ nanocomposite structures are studied, which integrates both electronic conductivity and buffering matrix design strategies. The $\mathrm{CNTs}-\mathrm{TiO}_{2}$ nanostructured electrodes have been prepared and applied as anode materials for lithium-ion batteries, which exhibit higher lithium storage capacities and better cycling performance compared to single CNTs and $\mathrm{TiO}_{2}$ electrodes.

\section{Experimental details}

The multiwall carbon nanotubes (MWCNT) employed in this work were supplied by Arry International (Ger-

*corresponding author; e-mail: guler@sakarya.edu.tr many). The nanotubes were synthesized by catalytic carbon vapour deposition (CCVD) and had a purity of around $90 \%$. The CNT diameter ranged between 10 and $20 \mathrm{~nm}$. Purification and chemical oxidation of MWCNTs $[5,6]$ was carried out with different oxidation agents supplied by Aldrich. Concerning the fabrication of MWCNT buckypapers, stable aqueous CNT suspensions at a concentration of $1 \mathrm{mg} / \mathrm{ml}$ were prepared by tip sonication for $60 \mathrm{~min}$. These dispersions were then vacuum filtered through polycarbonate membrane filters of $450 \mathrm{~nm}$ pore size. The average thickness of the produced buckypapers is approximately $100 \mu \mathrm{m}$ and their diameter about $16 \mathrm{~mm}$.

The coating and oxidation processes have been performed in a multifunctional magnetron sputtering PVD unit equipped with thermal evaporation, dc and rf units. Nanostructured $\mathrm{TiO}_{2}$ thin films were manufactured in two steps. In the first step, pure metallic titanium is coated onto CNT mats via thermal evaporation by using high purity metallic titanium. In the second step, nanostructured $\mathrm{TiO}_{2}$ thin films were obtained via DC plasma oxidation process. Plasma oxidations of the titanium films were carried out at high purity oxygen $(99.999 \%)$ and argon $(99.9999 \%)$ gas mixture in a ratio of $1: 1$. The oxidation time and total chamber pressure for each oxidation condition was kept constant at 5 min and $1 \mathrm{~Pa}$, respectively. $75 \mathrm{~W}, 100 \mathrm{~W}$ and $125 \mathrm{~W}$ dc powers were chosen for plasma oxidation and the effect of dc power on the titanium dioxide formation and battery performance was investigated. The phase structures of the deposited films were investigated by X-ray diffraction (XRD) (Rigaku D/MAX 2000 with multipurpose attachment) with $\mathrm{Cu} K_{\alpha}$ radiation. The grain size of the thin films was calculated from the Scherrer formula [6];

$$
D=\frac{0.9 \lambda}{B \cos \theta}
$$

where $D$ is the mean grain size, $\lambda$ is the $\mathrm{X}$-ray wavelength, $B$ is the corrected full-width at half maximum (FWHM) and $\theta$ is the Bragg angle.

The morphology was observed by scanning electron microscopy (SEM) (JSM-6060 LV system). Coin-type 
(CR2016) test cells were assembled in an argon-filled glove box, directly using the $\mathrm{TiO}_{2}$ coated bukypaper substrates as the working electrode, a lithium metal foil as the counter electrode, a micro porous polypropylene (PP) membrane (Cellgard 2400) as the separator, and $1 \mathrm{M}$ solution of $\mathrm{LiPF}_{6}$ in ethylene carbonate (EC) and dimethyl carbonate (DMC) (1:1 by weight) as the electrolyte. The cells were cyclically tested on a MTI Model BST8-MA electrochemical analyzer using $1 \mathrm{C}$ current density over a voltage range of $1-3 \mathrm{~V}$.

\section{Results and discussions}

buckypapers prepared by vacuum filtration of well-dispersed CNT aqueous suspensions were found to be uniform, smooth and crack-free disks exhibiting significant structural integrity, as confirmed by SEM images (Fig. 1). The produced thin films consist of randomly interconnected CNTs forming a porous structure as it is apparent from Fig. 1a. The SEM image of a cross-section
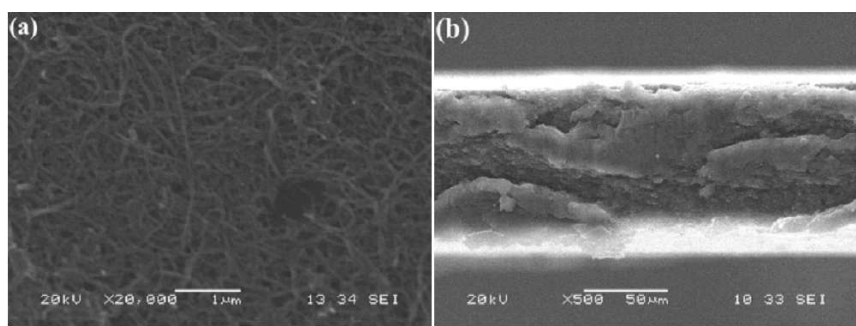

Fig. 1. Typical SEM images of (a) surface and (b) cross-section area for the buckypaper.

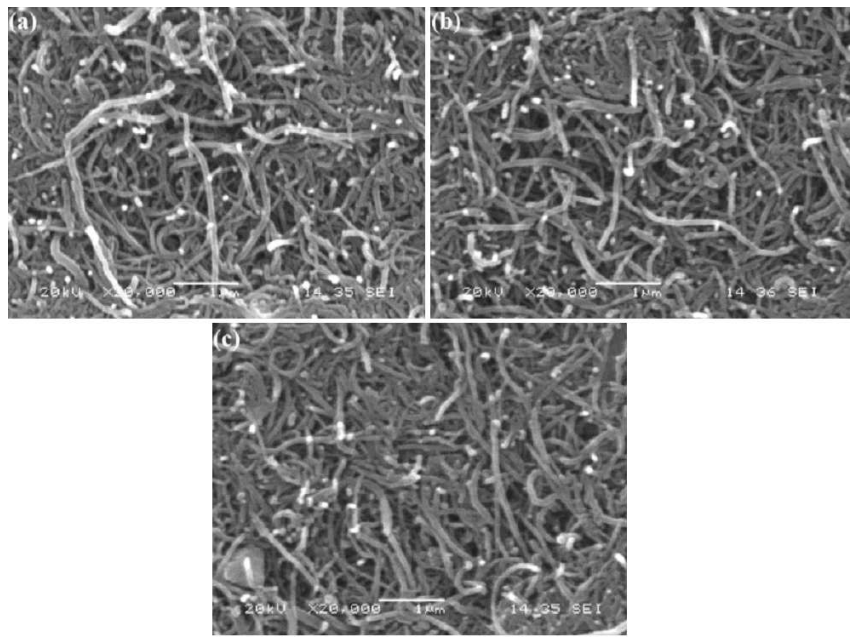

Fig. 2. SEM images of the films oxidized by (a) $75 \mathrm{~W}$ $\mathrm{dc}$, (b) $100 \mathrm{~W}$ dc and (c) $125 \mathrm{~W}$ dc powers.

of a representative sheet (Fig. 1b) indicates an almost homogeneous CNT deposition through the thickness giving rise to a dense morphology.
The surfaces of the coated buckypapers were shown in Fig. $2 \mathrm{a}-\mathrm{c}$, respectively. The surfaces of the coated buckypapers were found to be smooth and uniformly coated with $\mathrm{TiO}_{2}$. The volume of the CNTs was increased significantly after deposition process.

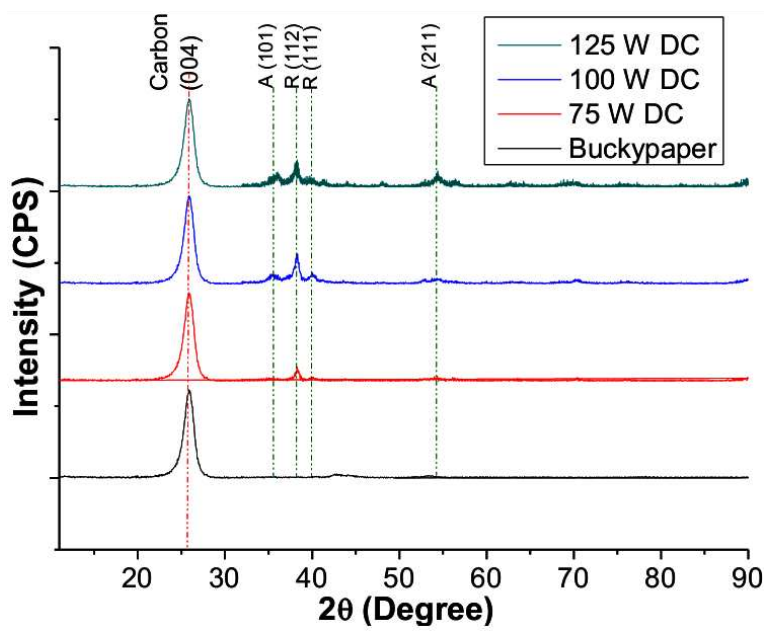

Fig. 3. XRD patterns of produced MWCNT based buckypaper and $\mathrm{TiO}_{2}$ coatings at various oxidizing powers.
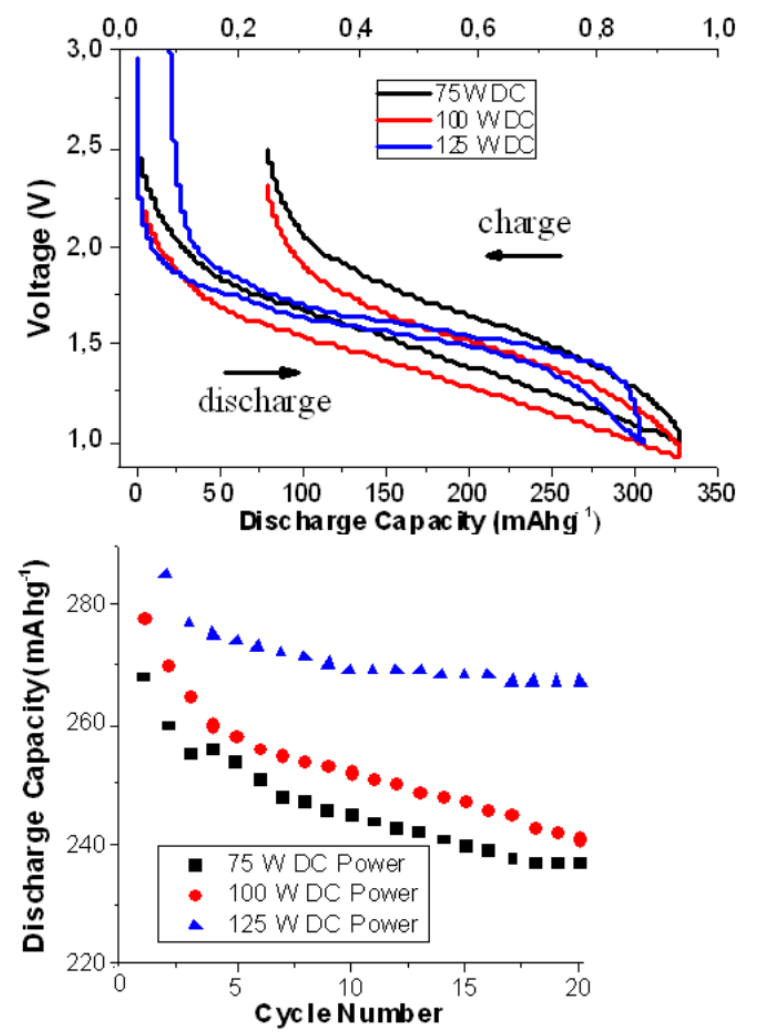

Fig. 4. Galvanostatic charge/discharge patterns of produced MWCNT based buckypaper and $\mathrm{TiO}_{2}$ coatings at various oxidizing powers. 
Figure 3 shows the X-ray diffraction pattern of the MWCNT based buckypaper, as-deposited and oxidized films. All reflexes were assigned to carbon (JCPDS 026-1080), $\mathrm{TiO}_{2}$ anatase (JCPDS 21-1272) and rutile (JCPDS 21-1276). The grain sizes of the films were calculated via Scherrer's formula and were found to increase with the decrease of oxidation power. The calculated values are $3.23 \mathrm{~nm}, 9.25 \mathrm{~nm}$, and $16.4 \mathrm{~nm}$ for the films oxidized at $75 \mathrm{~W}, 100 \mathrm{~W}$ and $125 \mathrm{~W}$, respectively.

The galvanostatic discharge-charge curves at current density $33.5 \mathrm{~mA} \mathrm{~g}^{-1}(1 \mathrm{C})$ of nanosized rutile and anatase $\mathrm{TiO}_{2}$ between 1 and $3 \mathrm{~V}$ vs. $\mathrm{Li}^{+} / \mathrm{Li}$ is presented in Fig. $4 \mathrm{a}$. As could be seen from the figures, the number of active $\mathrm{Li}$ ions was increased via increasing oxidation power. Figure $4 \mathrm{~b}$ shows the cycling performance of the films oxidized by using $75 \mathrm{~W}, 100 \mathrm{~W}$, and $125 \mathrm{~W}$ dc powers in the potential range of $3-1 \mathrm{~V}$. The capacity fading ratios for the films deposited by using $75 \mathrm{~W}, 100 \mathrm{~W}$, and $125 \mathrm{~W}$ are $9 \%, 11 \%$, and $13 \%$ after 20 cycles, respectively. The capacity retention is also stabilized after 17 th cycle and no capacity fading was observed. We conclude that the oxidation by using $125 \mathrm{~W}$ dc power contributes more to the enhancement of capacity retention due to smaller crystal size and larger surface area.

\section{Conclusions}

Carbon nanotube thin sheets - buckypapers - were prepared from multi-walled carbon nanotubes oxidized with different oxidation agents. Nanocrystalline $\mathrm{TiO}_{2}$ thin films were deposited onto buckypaper substrates via thermal evaporation followed by in situ plasma oxidation under different oxidation powers as a possible anode material for lithium ion batteries. The films oxidized by using $125 \mathrm{~W}$ dc power exhibited higher initial capacity and better cycling stability than the films oxidized via $75 \mathrm{~W}$ and $100 \mathrm{~W}$ dc powers.

\section{Acknowledgments}

This work is supported by the Scientific and Technological Research Council of Turkey (TUBITAK) under the contract number 109M464. The authors thank the TUBITAK MAG workers for their financial support.

\section{References}

[1] P.G. Bruce, B. Scrosati, J.M. Tarascon, Angew. Chem. Int. Ed. 47, 2930 (2008)

[2] E. Serrano, G. Rus, J. Garcia-Martinez, Renew. Sust. Energy Rev. 13, 2373 (2009).

[3] J. Chen, F. Cheng, Acc. Chem. Res. 42, 713 (2009).

[4] S. Iijima, Nature 354, 56 (1991)

[5] B. Vigolo, A. Penicaud, C. Coulon, C. Sauder, R. Pailler, C. Journet, P. Bernier, Science 290, 1331 (2000).

[6] M.E. Itkis, F. Borondics, A. Yu, R.C. Haddon, Nano Lett. 7, 900 (2007). 